\title{
mTOR signalling: the molecular interface connecting metabolic stress, aging and cardiovascular diseases
}

\author{
Z. Yang and X-F. Ming
}

Laboratory of Vascular Biology, Division of Physiology, Department of Medicine, Faculty of Science, University of Fribourg, Fribourg, Switzerland

Address for correspondence: Professor Z Yang, Laboratory of Vascular Biology, Department of Medicine, University of Fribourg, Rue du Musée 5, CH-1700 Fribourg, Switzerland.

E-mail: zhihong.yang@unifr.ch

\begin{abstract}
Summary
The continuing increase in the prevalence of obesity and metabolic disorders such as type-II diabetes and an accelerating aging population globally will remain the major contributors to cardiovascular mortality and morbidity in the 21 st century. It is well known that aging is highly associated with metabolic and cardiovascular diseases. Growing evidence also shows that obesity and metabolic diseases accelerate aging process. Studies in experimental animal models demonstrate similarity of metabolic and cardiovascular phenotypes in metabolic diseases and old age, e.g. insulin resistance, oxidative stress, chronic low grade inflammation, cardiac hypertrophy, cardiac fibrosis, and heart failure, as well as vascular dysfunctions. Despite intensive research, the molecular mechanisms linking metabolic stress, aging, and ultimately cardiovascular diseases are still elusive. Although the mammalian target of rapamycin (mTOR) signalling is a well known regulator of metabolism and lifespan in model organisms, its central role in linking metabolic stress, aging and cardiovascular diseases is recently emerging. In this article, we review the evidence supporting the role of mTOR signalling as a molecular interface connecting metabolic stress, aging and cardiovascular diseases. The therapeutic potentials of targeting mTOR signalling to protect against metabolic and age-associated cardiovascular diseases are discussed.
\end{abstract}

Keywords: Aging, cardiovascular diseases, mTOR, obesity.

\section{Introduction}

The increasing burden of obesity and associated metabolic disorders and an accelerating aging population globally are predicted to become the great challenges for our society in this century $(1,2)$. Aging is highly associated with obesity and metabolic disorders such as type-II diabetes as well as cardiovascular disease which is one of the leading causes of death (3). Evidence is emerging in recent years that obesity and associated metabolic disorders promote cellular and organism aging $(4,5)$, which reduces lifespan and particularly the healthy lifespan, the living period free of diseases. There is compelling evidence showing that oxidative stress and inflammation are the common mechanisms of meta- bolic disorders associated with obesity, aging and cardiovascular diseases $(6,7)$. However, the molecular pathways that connect metabolic disorders, aging and cardiovascular diseases are largely elusive.

Early studies provided convincing evidence for a potential role of the target of rapamycin (TOR) signalling pathway in longevity regulation in invertebrates (8), which has been recently extended to rodent mice (8). A role of deregulated mammalian TOR (mTOR), also named by some authors as 'mechanistic TOR' signalling, has also been shown to be involved in obesity and associated metabolic disorders (9). Only limited information is available on the role of mTOR in metabolic and age-associated cardiovascular diseases. The primary emphasis of this review 
article is to discuss the emerging evidence for the role of mTOR signalling as the molecular interface connecting metabolic diseases, aging and cardiovascular diseases.

\section{Regulation of mTOR signalling pathways}

mTOR belongs to the phosphatidylinositol 3-kinase (PI3K)-related kinase family with a molecular weight of $\sim 289 \mathrm{kDa}(10)$. It serves as an intracellular sensor for energy metabolism, nutrient availability, and stresses, regulating cellular and organism growth and metabolism to adapt the environmental changes. Dys-regulation of mTOR signalling, therefore, significantly impacts a wide spectrum of physiological functions and disease processes in organisms (10). The detailed molecular signalling network and regulation of mTOR signalling have been reviewed comprehensively in several articles (8-10). Here, we summarize a few important biochemical features of mTOR signalling network (Fig. 1).

\section{mTOR complexes}

mTOR, with other molecular components, forms two distinct complexes, mTOR complex 1 (mTORC1) and mTOR complex 2 (mTORC2), in which mTOR enzymatic activity is regulated by its unique accessory proteins raptor and rictor, respectively. Raptor and rictor function as scaffold proteins to recruit substrates and regulators in the respective complex.

\section{Sensitivity to the immunosuppressant rapamycin}

Rapamycin inhibits preferentially mTORC1, but chronic treatment with rapamycin in vitro and in vivo inhibits both mTORC1 and mTORC2 $(11,12)$.

\section{The upstream regulators}

mTORC1 activity is regulated by multiple factors including growth factors, stressors such as DNA damage, hypoxia,
Figure 1 Major distinct characteristics of the two known mTOR signalling complexes, mTORC1 and mTORC2, in composition, rapamycin sensitivity, upstream signals, substrates and biological functions. Adapted from Laplante and Sabatini (10). 4EBP1, elF4E-binding protein 1; AMPK, AMP kinase; Atg, autophagy related; DEPTOR, DEP domain containing mTOR-interacting protein FIP200, 200 kDa FAK family

kinase-interacting protein; FOXO, forkhead box protein $\mathrm{O}$; $\mathrm{mLST8}$, mammalian lethal with sec-13 protein 8 ; $m \sin 1$, mammalian stress-activated map kinase-interacting protein 1; PPAR $\gamma$, peroxisome

proliferator-activated receptor- $\gamma$; PRAS40, proline-rich Akt substrate $40 \mathrm{kDa}$; protor, protein observed with rictor; raptor, regulatory-associated protein of mammalian target of rapamycin; REDD1, regulated in development and DNA damage response 1; rictor, rapamycin-insensitive companion of mTOR; Rom2, Rho1 GDP-GTP exchange protein 2; SGK, serum- and glucocorticoid-regulated kinase; SREBP, sterol regulatory element-binding protein; Tel2, telomere maintenance 2; TSC, the tuberous sclerosis.

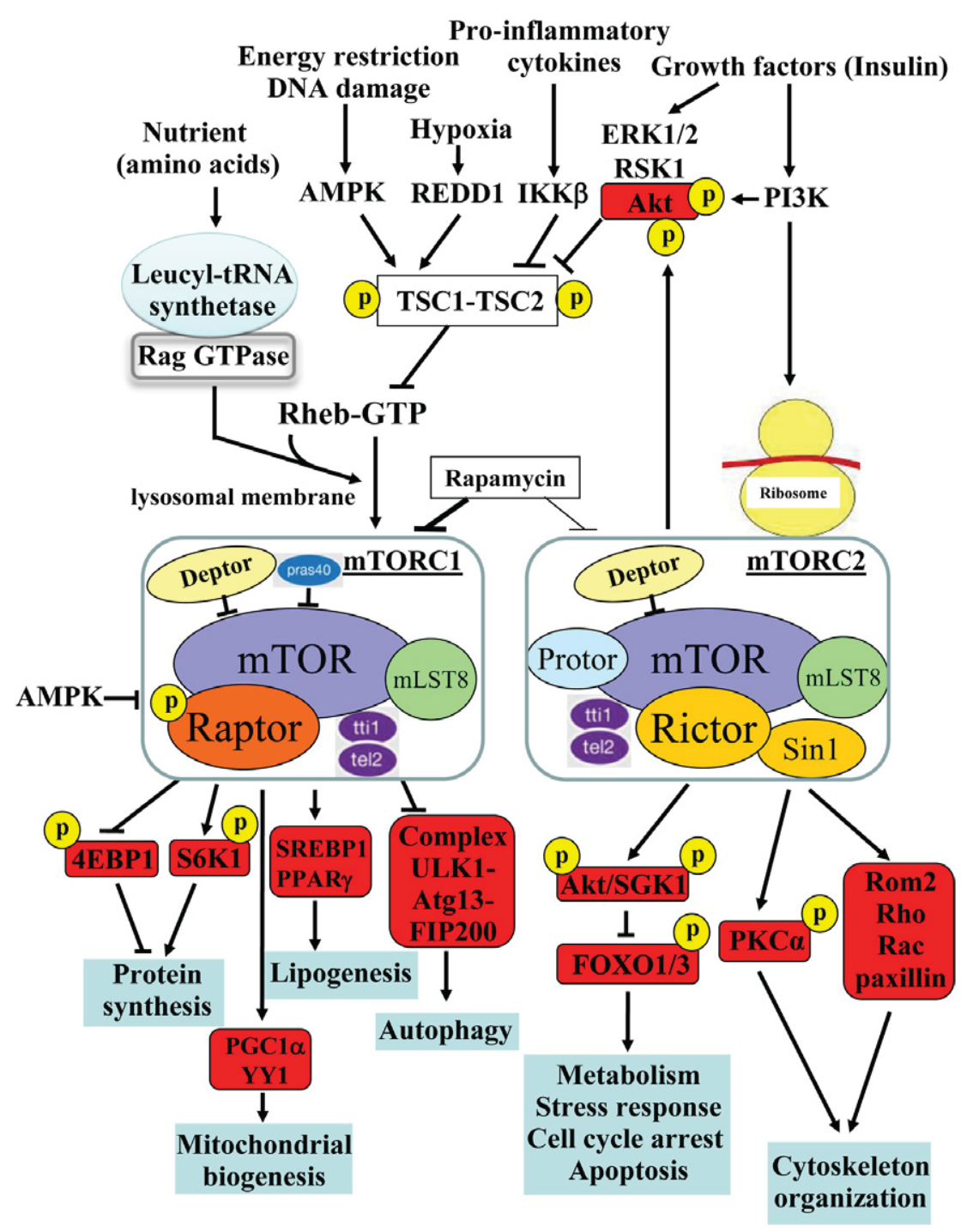


and nutrients, while mTORC2 seems to be regulated only by growth factors such as insulin. While the mechanisms of mTORC1 activation are intensively investigated, the mechanisms of mTORC2 activation are less well characterized. A recent study shows that growth factors activate mTORC2 through interaction with ribosomes in response to PI3K stimulated by growth factors such as insulin (13). For activation of mTORC1, the small GTPase protein Rheb is the most proximal molecule directly activating mTORC1 (10). Importantly, Rheb activity is negatively regulated by the TSC1/2, the GTPase activating protein which modulates Rheb by converting the active Rheb-GTP into the inactive Rheb-GDP and thus reduces mTORC1 activity. Many growth factors phosphorylate and inactivate TSC1/2 via Akt and ERK1/2 kinases, leading to activation of Rheb-mTORC1 pathway. In contrast, energy restriction, DNA damage or hypoxia activates TSC1/2 through activation of AMPK or REDD1, respectively, resulting in inactivation of Rheb and inhibition of mTORC1 (Fig. 1). There are studies indicating that AMPK inhibits mTORC1 activity through phosphorylation of raptor, resulting in inhibition of mTORC1, which is independent of TSC1/2 (9) (Fig. 1). The nutrients amino acids, however, activate mTORC1 specifically through Rag GTPases, which is independent of TSC1/2. In the presence of amino acids, Rag GTPases interact with mTORC1 and translocate the complex from cytoplasm to lysosomal membranes where it is activated by Rheb (10). Two research groups, respectively, in yeast and mammalian cells, have recently characterized that the Leucyl-tRNA synthetase exerts a non-canonical role in amino acid-induced mTORC1 activation $(14,15)$. In the presence of amino acids, the LeucyltRNA synthetase interacts directly with Rag leading to the formation of RagA/B-RagC/D complex in their correct GTP/GDP-loading status (RagA/B-GTP and RagC/DGDP), enabling it to directly interact with raptor component of mTORC1 and the localization of mTORC1 to lysosomal membrane. Therefore, the Leucyl-tRNA synthetase is an intracellular amino acid sensor for amino acid-induced mTORC1 activation (Fig. 1).

\section{The downstream effectors}

mTORC1 enhances protein synthesis through S6K1 and eIF-4E-binding protein 1 (4E-BP1), which is the best characterized function of mTORC1 signalling. Upon phosphorylation by mTORC1, 4E-BP1 dissociates from eIF4E, relieving its suppressing effect on mRNA translation, while S6K1, when phosphorylated by mTORC1, promotes mRNA translation. mTORC1 also activates the transcription factors SREBP1 and PPAR $\gamma$, inducing lipogenesis in the liver, promotes mitochondrial biogenesis and oxidative metabolism by activating PGC1 $\alpha / Y Y 1$ (reviewed in (10)). It inhibits autophagy through phosphorylation of the
ULK1-Atg13-FIP200 complex and also through regulation of lysosomal function (16), a central mechanism that degrades and recycles damaged organelles in the cells, allowing the cells to adapt to the condition of nutrient deprivation. mTORC2 exerts its effects on metabolism, stress responses, apoptosis, and cytoskeleton organization through phosphorylation of many AGC kinases including Akt, serum- and glucocorticoid-induced protein kinase (SGK), protein kinase C- $\alpha$ (PKC $\alpha)$ and Rho1 GDP-GTP exchange protein-2 (Rom2) (10). Since mTORC2 activates Akt that in turn enhances mTORC1 activity through inactivation of TSC1/2, mTORC2 is the upstream of mTORC1 upon stimulation by growth factors (Fig. 1).

\section{mTOR signalling in metabolic disorders}

Early studies focused much more on the role of mTORC1 signalling in regulation of cell growth and protein synthesis. Its role in regulating metabolism has attracted much attention in recent years, together with the notion of intermittent vs. persistent signalling to explain its differential effects. A short-term activation of mTORC1 signalling during intermittent availability of nutrients within a physiological range is necessary for anabolic metabolism, energy storage, and consumption, as well as normal cell/tissue development. This is confirmed by the fact that mice defective in muscle mTORC1 activity have reduced muscle mass, decreased PGC-1 $\alpha$-mediated mitochondrial oxidative metabolism, and die early (17). However, under conditions of overfeeding or chronically high availability of nutrients including glucose and amino acids, a persistent activation of mTORC1 signalling occurs $(18,19)$ (Fig. 2). Indeed, evidence demonstrates increased basal mTORC1 activity in both genetic and diet-induced animal models of obesity and metabolic disorders in metabolically active organs/tissues such as liver (19), skeletal muscle $(19,20)$, adipose tissue $(18,21)$, as well as vasculature $(5)$ and heart $(22,23)$. In addition, in obesity and metabolic syndrome, insulin resistance and hyperinsulinaemia occur. The enhanced insulin levels further stimulate mitogenic pathways (i.e. p44/ 42mapk-mTORC1) while decreasing metabolic pathways (i.e. the Akt) resulting from hyperactive S6K1-mediated phosphorylation of insulin receptor substrate 1 (IRS-1) at serine residues which promotes IRS1 degradation (18) (Fig. 2). mTORC1 has been shown to play a key role in lipid storage by stimulating the synthesis of triglycerides in white adipose tissue and differentiation of preadipocytes into white adipocytes through up-regulation of PPAR- $\gamma$, a factor that up-regulates adipocyte-specific gene programme $(24,25)$. This is evidenced by the finding showing that adipocyte-specific deletion of raptor in mice leads to reduced white adipose tissue mass (26). Recent studies also implicate a role of mTORC2 in lipogenesis through activation of Akt-mTORC1 (27). Therefore, a chronic 


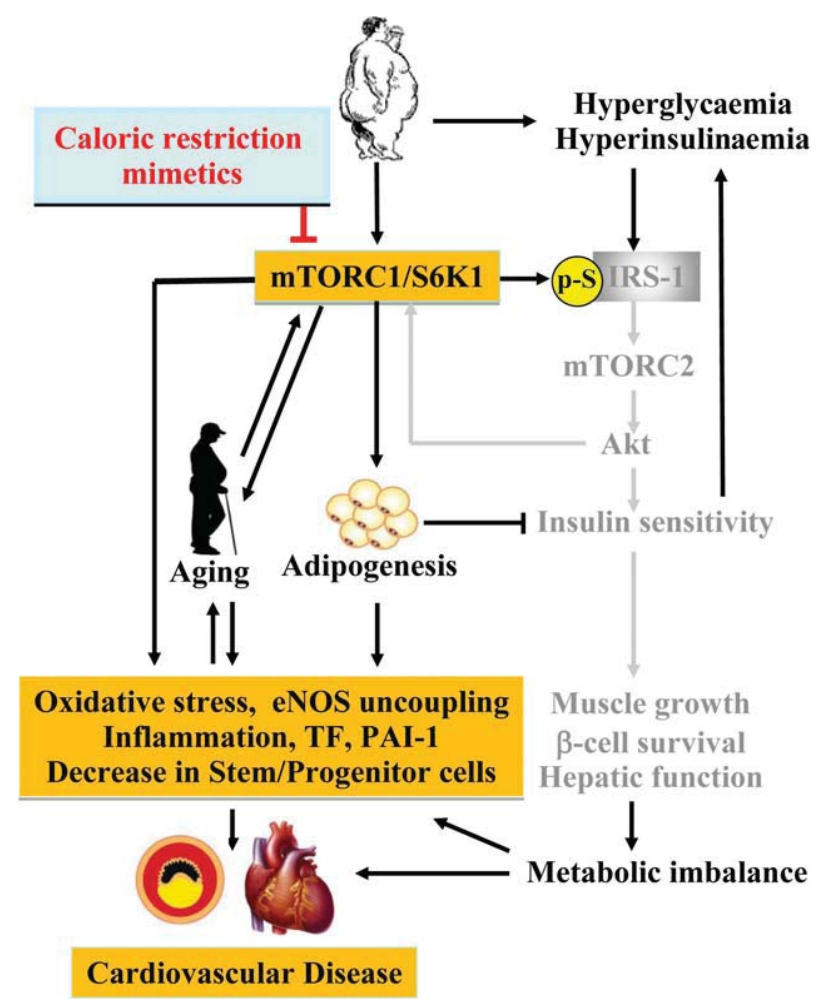

Figure 2 mTORC1/S6K1 signalling in cardiovascular diseases related to obesity and aging. Overnutrition leads to persistent hyperactive mTORC1/S6K1 signalling which phosphorylates IRS-1 at serine residues ( $\mathrm{p}-\mathrm{S}$ ), leading to reduction in insulin signalling (mTORC2/Akt) and decrease in insulin sensitivity, resulting in hyperglycaemia and hyperinsulinaemia as well as metabolic imbalance via influencing muscle growth, $\beta$-cell survival and hepatic function. The hyperactive mTORC1/S6K1 promotes aging (directly or indirectly) and adipogenesis, which further aggravates obesity and insulin resistance, resulting in oxidative stress, eNOS uncoupling, inflammation, pro-coagulation factor production, and decreased stem/progenitor cell repairing capacity, and ultimately cardiovascular diseases. Fading part of the picture represents reduced activity. Caloric restriction mimetics inhibit mTORC1/S6K1 signalling. PAI-1, plasminogen activator inhibitor-1; TF, tissue factor.

mTORC1 activation contributes to obesity by enhancing excess fat deposition in white adipose tissue, liver and muscle, which in turn promotes insulin resistance, leading to reduced glucose uptake and glycogen synthesis in the liver and muscle, and increased gluconeogenesis and glucose release by the liver, further exacerbating hyperglycaemia and hyperinsulinaemia that are generated by excess nutrients (Fig. 2). Because of insulin resistance under the condition of hyperactive mTORC1 signalling, a decreased Akt activation unmasks activation of FOXO1 which leads to ubiquitin ligase expression, resulting in protein catabolism, so that the protein anabolic effect of mTORC1 under the overnutrition condition does not efficiently occur, resulting in muscle wasting (28). This mechanism may provide an explanation for the decreased muscle mass in obesity and aging, in which mTORC1 signalling is persistently high $(29,30)$. In line with these effects of mTORC1 signalling, mice deficient in the mTORC1 downstream effector S6K1 $\left(\mathrm{S} 6 \mathrm{~K}^{-1-}\right)$ are protected from insulin resistance and obesity under high-fat diet (HFD) feeding, further demonstrating the role of mTORC1-S6K1 in obesity, insulin resistance and metabolic diseases (18) (Fig. 2).

As mentioned above, mTORC1 signalling is required for multiple physiological functions of cells, tissues and organs: systemic mTOR knockout in mice is embryonic lethal (31) and systemic deficiency in S6K1 results in various abnormalities. For example, the $\mathrm{S} 6 \mathrm{~K} 1^{-/}$mice have reduced islets and decreased pancreatic insulin contents, and are therefore hypoinsulinemic and glucose intolerant (32). This is due to the fact that mTORC1-S6K1 signalling is necessary for growth of pancreatic $\beta$-cells in response to glucose and amino acids (32). On the other hand, persistent activation of mTORC1-S6K1 signalling under the condition of overnutrition leads to pancreatic $\beta$-cell apoptosis through phosphorylation and subsequent proteosomal degradation of IRS2 (33). Given that mTORC1-S6K1 signalling is also a critical pathway for normal skeletal muscle growth (17), fully inhibition of mTORC1-S6K1 signalling should not be the goal for therapeutic purposes to treat metabolic disorders.

\section{mTOR signalling in aging}

Evidence for a role of mTOR signalling in organism lifespan and aging has been comprehensively demonstrated in almost all model organisms including yeast, worms, flies and mice. Genetic inactivation or pharmacological inhibition of mTORC1 or its downstream effector kinase showed lifespan extension in these models (8). Mice treated with the mTORC1 inhibitor rapamycin starting at a late age show prolonged lifespan $(34,35)$, whereas mice treated with rapamycin starting early in life show reduction in age-associated learning and memory deficits. Rapamycin does not have the effects on cognition when the treatment starts at a later age (36), suggesting that mTORC1 signalling contributes to age-related decline in cognition but is not reversible once the brain damage has already occurred. Mice with down-regulation of insulin and insulin-like growth factor (IGF-1) signalling show reduced mTORC1S6K1 activity and increased lifespan $(37,38)$, which further support a role for mTORC1 in the aging process (Fig. 2). Interestingly, the effect of inhibiting mTORC1 signalling on longevity can be mimicked by caloric restriction across different species $(8,39)$. Although no firm evidence exists for the anti-aging effect of caloric restriction in humans, a recent study has shown that caloric restriction slows aging in rhesus monkeys (40). Moreover, it has been documented that the Okinawa populations who consume $20 \%$ less calories compared to the average caloric consumption of the 
Japanese population live longer and display lower incidences of cancer and cardiovascular diseases in the elderly (41). A small-scale caloric restriction study in adult humans over 6 years also demonstrates less cardiovascular risk factors including lower body mass index, body fat deposition, blood pressure, fasting plasma levels of glucose and insulin, lower blood markers of inflammation, and beneficial lipid profiles (42), and also shows better left ventricular diastolic function than healthy age- and sex-matched controls (43). Also, patients with type-II diabetes benefit from caloric restriction and show improvement of cardiac function (44). It remains to be determined whether the beneficial effects of caloric restriction in humans are mediated by inhibition of mTORC1-S6K1 signalling and whether the anti-aging effects could be translated to lifespan extension in healthy elderly subjects and in patients with metabolic disorders.

It seems that the two well known downstream effectors of mTORC1, i.e. S6K1 and 4EBP1, are involved in regulation of lifespan and aging. A reduction or deficiency in S6K1 activity increases lifespan in various species including mice which are also resistant to age-related pathologies (45). In flies, loss of 4E-BP reduces lifespan extension induced by dietary restriction, whereas overexpression of a gain-of-function form of 4E-BP is sufficient to extend lifespan under overnutrient supply conditions (46). The underlying molecular mechanisms are not clear. However, substantial evidence indicates that the induction of autophagy after inhibition of mTORC1 signalling degrades damaged proteins or organelles, and therefore plays a crucial role in reducing aging (47). Future work shall confirm this mechanism in higher organisms.

A recently emerging concept that remains to be confirmed is that mTORC1 accelerates aging by depletion of stem/progenitor cells (Fig. 2). Inhibition of mTORC1 signalling has been shown to slow depletion of stem/ progenitor cells and improve the functions of these cells (48). A recent study also shows that the mTORC1 signalling in haematopoietic stem cells (HSC) of old mice is higher than that of young mice and treatment of the old mice with rapamycin improves HSC self-renewal and haematopoietic function, improves immunity, and increases lifespan (49), thereby suggesting a role for mTORC1 signalling in accelerating aging through impaired repairing capacity of stem/progenitor cells.

Although there is compelling evidence demonstrating the role of mTORC1-S6K1 in regulation of lifespan extension, organism aging and metabolic stress induced by overnutrition in animal models, only little information is available about the role of mTORC1-S6K1 in cardiovascular diseases associated with these conditions. In the next sections, we will discuss the role and mechanisms of mTOR signalling in pathogenesis of cardiovascular diseases under the conditions of metabolic disorders and aging.

\section{Cardiovascular phenotype in metabolic disorders and aging}

The cardiovascular phenotypes in metabolic diseases and aging share many common characteristics and are accompanied by structural and functional changes in the heart and blood vessels. The heart in metabolic disorders and aging shows adaptation and maladaptation to alterations in lipid and/or glucose metabolism, accompanied by cardiac insulin resistance and ultimately leading to cardiac dysfunction and heart failure (for detailed description of metabolic changes of the heart in overnutrition, please see (50)). The heart from patients with metabolic disorders and in the elderly is more susceptible to myocardial ischemic injury (51,52). Moreover, the heart in metabolic diseases and aging exhibits cardiac hypertrophy, oxidative stress, interstitial fibrosis due to chronic deposition, and remodelling of extracellular matrix, leading to impaired diastolic function and heart failure $(53,54)$. A decreased regenerative capacity of cardiac stem cells, possibly due to impaired cell division and accelerated cell senescence, and an increased cardiac myocyte apoptosis may also contribute to cardiac dysfunction in metabolic diseases and in the elderly $(54,55)$. In obesity, increased perivascular adipose tissues surrounding coronary arteries and the associated adipose tissue inflammations and cardiac steatosis promote cardiac dysfunction and coronary heart disease (56). It is well demonstrated in the past years that obesity is associated with chronic low-grade inflammation manifested by adipose tissue pro-inflammatory macrophage infiltration with typical crown-like structure in adipose tissues, and increased local and systemic levels of inflammatory cytokines, contributing to insulin resistance and type-II diabetes (57). This phenotype of low-grade adipose tissue inflammation is also present in aging mice $(58,59)$. It is, however, not clear whether the age-associated adipose tissue inflammation is due to aging per se or age-associated insulin resistance and type-II diabetes.

The vascular phenotypes in metabolic diseases and aging also exhibit many pathological features in common. They are associated with increased arterial wall thickening and generalized vascular stiffness (60). At the cellular and molecular levels, oxidative stress, vascular inflammation, as well as endothelial cell and progenitor cell dysfunction (mainly reflected by decreased vasoprotective endothelial nitric oxide [NO] bioavailability) intertwine with each other, representing the major mechanisms leading to exaggerated atherosclerosis in pathological conditions and aging $(6,60)$. In metabolic disorders and aging, the endothelial expression of inflammatory adhesion molecules such as ICAM-1 and VCAM-1 is increased (6); these participate in the initiation and progression of atherosclerosis through enhanced monocyte-endothelial cell interaction. Elevated plasma concentration of sVCAM-1, sICAM-1, tissue factor 
(TF), and PAI-1, factors that are involved in inflammation and thrombosis, is observed in metabolic diseases and aging (6,61-63). Adipose tissue and endothelial cells have been shown to be the important sources for TF and PAI-1 production $(63,64)$. Importantly, obesity and aging have synergistic effects on TF and PAI-1 production (63) (Fig. 2), which may explain the increased incidence of thrombosis risks in patients with metabolic syndrome and in the elderly population $(65,66)$. Moreover, in elderly patients and in patients with diabetes, the number and the regenerative capacity of circulating endothelial progenitor cells are decreased (67,68), which implicates the impairment of cardiovascular repairing capacity after cardiac or vascular injury under these conditions. The underlying molecular mechanisms are, however, not clear. Evidence suggests a critical role of oxidative stress in the impairment of endothelial cell and progenitor cell function, manifested by eNOS dysfunction and vascular inflammation (69).

\section{Oxidative stress, eNOS uncoupling, and vascular inflammation in metabolic disorders and aging}

Increased oxidative stress in metabolic disorders and aging has been convincingly demonstrated by a wide range of experimental and clinical studies $(6,70,71)$. A variety of mechanisms/enzymes that contribute to oxidative stress in cardiovascular system in metabolic disorders and aging have been demonstrated; these include mitochondrial dysfunction, $\mathrm{NAD}(\mathrm{P}) \mathrm{H}$ oxidase, xantine oxidase, cyclooxygenase, lipoxygenase, etc. A detailed description of the role and source of the oxidative stress has recently been the subject of a comprehensive review article (71). Here, we would like to focus on the role of 'eNOS uncoupling' as the potential mechanism of oxidative stress in metabolic diseases and aging. The endothelial NO, which is produced via eNOS from L-arginine in the presence of the cofactor $\mathrm{BH}_{4}$, plays a protective role against cardiovascular diseases through multiple functions: NO causes vascular smooth muscle relaxation, inhibits platelet aggregation and leukocyte adhesion, and prevents endothelial cells from apoptosis and senescence (72). eNOS dysfunction or deficiency has been shown to contribute to insulin resistance, accelerated atherosclerosis, cardiac dysfunction with aging, ultimately a significantly shorter lifespan in mice (73-75). Although decreased eNOS gene expression has been reported to be responsible for endothelial dysfunction under various conditions, including atherosclerosis, metabolic diseases, and aging (72), decreased eNOS enzymatic dysfunction accompanied by increased eNOS gene expression is usually observed under these conditions (76-78). The results suggest that endothelial dysfunction in metabolic diseases and aging is mainly attributed to eNOS enzymatic dysfunc- tion rather than to decreased eNOS gene expression, at least in the early stages of the diseases.

Indeed, recent studies provide increasing evidence demonstrating that eNOS uncoupling is a crucial mechanism contributing to oxidative stress in metabolic diseases and aging as well as in atherosclerosis (78-80). Under the condition of eNOS uncoupling, eNOS produces increasing amount of $\mathrm{O}_{2}^{-}$instead of $\mathrm{NO}(78,81)$ usually associated with decreased eNOS dimer/monomer ratio $(78,81)$. Although the molecular mechanisms of eNOS uncoupling remain incompletely understood, the following mechanisms have been proposed:

(a) $\mathrm{BH}_{4}$ deficiency: the normal function of eNOS requires homodimerization of the enzyme which is stabilized by the cofactor $\mathrm{BH}_{4}$. The eNOS reductase domain generates electron flow from NADPH through FAD and FMN flavins, which are then transferred to the oxidase domain of other monomers in which L-arginine is metabolized to $\mathrm{NO}$ at the haem group in the active site (81). $\mathrm{BH}_{4}$ deficiency due to either defective synthesis or oxidative inactivation decreases eNOS dimer/monomer ratio and the catalytic activity of eNOS becomes uncoupled - that is uncoupling of NADPH oxidation and NO synthesis, with oxygen instead of L-arginine as terminal electron acceptor, resulting in $\mathrm{O}_{2}^{-}$generation (81);

(b) Limited specific pool of intracellular L-arginine bioavailability due to enhanced arginase activity (82) or production of endogenous eNOS inhibitor asymmetric dimethylarginine (83);

(c) The eNOS S-glutathionylation level is increased in spontaneously hypertensive rats, a metabolic syndrome model, as compared to normotensive animals accompanied with impaired endothelium-dependent relaxations that can be reversed after the S-glutathionylation of eNOS is removed by thiol-specific reducing agents $(84)$. Whether S-glutathionylation of eNOS also plays a role in endothelial dysfunction in other metabolic disease models or conditions and aging remains to be explored.

\section{mTOR signalling in eNOS uncoupling and vascular inflammation in metabolic diseases and aging}

As discussed above, the physiological role of the mTOR signalling is to support cell growth and survival. This concept is supported by studies showing that ablation of mTORC1 signalling is detrimental to cardiac tissue in chronic cardiac remodelling models $(85,86)$. However, a persistent uncontrolled activation of $\mathrm{mTOR}$ signalling does more harm than good. In the metabolic syndrome model of spontaneously hypertensive rats, enhanced mTORC1S6K1 signalling has been observed in the heart, contributing to the cardiac hypertrophy (87), which is thought being 
due to decreased AMPK activity, the upstream inhibitor of mTORC1 (88) (Fig. 1). In the left ventricles of hyperinsulinaemic Zucker Obese rats, mTORC1 signalling is also elevated, which could be explained by the hyperinsulinaemia or other hormonal factors such as angiotensin II or overnutrition, leading to selective activation of mTORC1 pathway $(89,90)$. It seems that there are dynamic profiles of mTORC1 signalling during the disease development. In the overt diabetes of ZDF rats, activation of mTORC1 signalling in the heart seems to be decreased to the normal level, which could be due to the development of pancreatic insulin deficiency at this stage (50). In HFD-induced obesity and metabolic syndrome mouse model, cardiac mTORC1 activity is increased and remained elevated during ischaemia, which is associated with suppressed autophagy in the heart (91). Importantly, treatment of the mice with rapamycin significantly enhances autophagy and reduces ischaemic myocardial infarction size in the mice on HFD. Similarly, genetic or pharmacological inhibition of S6K1 in the mouse model of myocardial infarction significantly improves cardiac function, and reduces cardiomyocyte apoptosis and fibrotic area (92). These studies underscore the critical role of mTORC1-S6K1 signalling in negative cardiac remodelling induced by cardiac ischaemic injury in the presence or absence of metabolic syndrome. Similar to mammals, Drosophila-fed HFD develops obesity and severe cardiac dysfunction, and genetic suppression of the mTOR signalling pathway prevents obesity and protects the flies against HFD-induced cardiac defects (93). These results further suggest that dys-regulation of mTOR activity mediates the deleterious effect of obesity on heart function (Fig. 2).

In the vasculature, increased mTOR signalling has also been observed in the aortas of mice fed with HFD, which is associated with increased vascular senescence and vascular dysfunction and more prone to peripheral and cerebral ischaemia, which is reduced by rapamycin treatment (5). This study provides the first evidence suggesting that mTOR signalling links obesity to vascular aging (Fig. 2). In line with the results of this study, a persistent hyperactive S6K1 activity in cultured senescent endothelial cells and in aortas of the old rats has been observed (78). The hyperactive S6K1 in senescent endothelial cells and aged rat aortas is accompanied with increased $\mathrm{O}_{2}^{-}$production derived from eNOS uncoupling (78). The causal role of S6K1 in eNOS uncoupling in cell senescence is demonstrated by the fact that overexpression of a S6K1 active mutant in young endothelial cells causes eNOS uncoupling, decreases eNOS dimer/monomer ratio, and accelerates endothelial senescence, and furthermore inhibition of mTORC1-S6K1 pathway either with rapamycin or by S6K1 silencing recouples eNOS function, i.e. improves NO production and inhibits $\mathrm{O}_{2}^{-}$production in the senescent cells and aortas of old rats (78). Interestingly, the naturally occurring polyphenol resveratrol, an intensively investigated antiaging, anti-oxidative, and anti-inflammatory compound $(94,95)$, is able to recouple eNOS in aging through inhibition of mTORC1-S6K1 signalling (78). It is most likely that resveratrol inhibits mTORC1-S6K1 signalling through inhibition of mTORC2, since the hyperactive Akt, the downstream effector kinase of mTORC2 and the upstream signalling of mTORC1-S6K1, is observed in senescent endothelial cells and resveratrol is able to normalize Akt activity in the cells (78). Our finding is consistent with the report that a hyperactive Akt plays a role in endothelial cell senescence (96). Further studies are required to establish a role of mTORC2 in endothelial senescence, metabolic diseases, and associated cardiovascular complications, and how resveratrol intervenes with mTORC2 activity leading to activation of Akt-mTORC1-S6K1 pathway. Also, the question remains whether enhanced mTOR signalling in obesity indeed plays a causal role in obesity-associated cardiovascular aging or vice versa.

There is considerable evidence showing that mTORC1 is also involved in endothelial TF expression and adhesion molecule VCAM-1 and ICAM-1 expression, which would contribute to the vascular inflammatory responses and thrombosis formation (Fig. 2). Numerous studies demonstrate that rapamycin or silencing mTOR enhances TF expression in endothelial cells $(97,98)$. However, silencing S6K1 reduces TF protein level in endothelial cells in response to thrombin or TNF- $\alpha$ without affecting TF mRNA expression. Conversely, overexpression of a constitutively active S6K1 mutant enhances TF protein level even in the mTOR-silenced cells (98). The results reveal an opposing effect of mTOR and S6K1 on endothelial TF expression and are best explained by the mechanisms that the markedly enhanced TF mRNA expression under the condition of mTORC1 inhibition is translated by S6K1-independent pathways such as RhoA, NF- $\mathrm{BB}$, and p38mapk (98), since blockade of RhoA, NF-kB and p38mapk either pharmacologically or genetically is able to reduce the up-regulation of TF protein level. These findings may have potential clinical implications. TF is highly expressed in cells within atherosclerotic plaques (99) and can be released into circulation after coronary intervention (100), which favours thrombus formation. With rapamycin (sirolimus)-eluting stents, TF expression in the vasculature and release from vascular cells including endothelial cells, smooth muscle cells and macrophages might be exaggerated because of the stimulating effect of TF expression by the mTORC1 inhibitor. This effect of rapamycin may increase thrombotic risk, despite its property of preventing vascular restenosis in patients with coronary artery disease (101), although inhibition of endothelial regeneration and in turn endothelialization of stent surface by drug-eluting stents may play a more prominent role in stent thrombosis (102). Whether specific S6K1 inhibitors are able to prevent 
vascular restenosis as the mTORC1 inhibitor rapamycin, and whether they are superior in reducing thrombotic propensity particularly in high-risk individuals such as patients with diabetes and elderly patients, are interesting topics of future investigations. Moreover, silencing S6K1 in endothelial cells is able to prevent E-selectin expression induced by TNF- $\alpha$ (103), implicating a possible role of S6K1 in regulation of endothelial-leukocyte interaction. Interestingly, high-glucose-induced VCAM-1 and ICAM-1 expression in endothelial cells can be inhibited by resveratrol (95). Whether this is due to inhibition of mTORC1-S6K1 signalling remains to be investigated. Further studies shall also examine the role of mTORC1-S6K1 in TF as well as adhesion molecule expression in metabolic diseases and aging in vivo.

\section{Conclusions}

Substantial evidence demonstrates that a sustained hyperactive mTOR signalling plays a crucial role in connecting metabolic stress, aging, and cardiovascular diseases through stimulation of oxidative stress and inflammatory responses (integratively summarized in Fig. 2). One of the important issues is to investigate whether targeting S6K1 directly with specific inhibitors (92) is a better approach than targeting mTORC1 in metabolic diseases, aging and associated cardiovascular diseases. Moreover, how much of the beneficial effects of resveratrol and Sirt1 activators are attributable to inhibition of mTORC1-S6K1 signalling either directly or indirectly should be investigated. Nevertheless, targeting mTOR signalling by newly developed caloric restriction mimetics such as rapamycin analogues or resveratrol derivatives may show less adverse effects and hold great promise for health benefits, i.e. improvement of metabolic profiles in metabolic diseases, deceleration of aging process, and improvement of cardiovascular functions, ultimately leading to extension of healthy lifespan.

\section{Conflict of interest statement}

The authors declare no conflict of interest.

\section{Acknowledgements}

The original own studies cited by this article were supported by the Swiss National Science Foundation (310000120435 and 310030-141070/1) and the Swiss Heart Foundation.

\section{References}

1. Scully T. Diabetes in numbers. Nature 2012; 485: S2-S3.

2. Christensen K, Doblhammer G, Rau R, Vaupel JW. Ageing populations: the challenges ahead. Lancet 2009; 374: 1196-1208.
3. Fontana L. Modulating human aging and age-associated diseases. Biochim Biophys Acta 2009; 1790: 1133-1138.

4. Minamino T, Orimo M, Shimizu I et al. A crucial role for adipose tissue p53 in the regulation of insulin resistance. Nat Med 2009; 15: 1082-1087.

5. Wang CY, Kim HH, Hiroi Y et al. Obesity increases vascular senescence and susceptibility to ischemic injury through chronic activation of Akt and mTOR. Sci Signal 2009; 2: ra11.

6. Ungvari Z, Kaley G, de Cabo R, Sonntag WE, Csiszar A. Mechanisms of vascular aging: new perspectives. J Gerontol A Biol Sci Med Sci 2010; 65: 1028-1041.

7. Hulsmans M, De Keyzer D, Holvoet P. MicroRNAs regulating oxidative stress and inflammation in relation to obesity and atherosclerosis. FASEB J 2011; 25: 2515-2527.

8. Evans DS, Kapahi P, Hsueh WC, Kockel L. TOR signaling never gets old: aging, longevity and TORC1 activity. Ageing Res Rev 2011; 10: 225-237.

9. Inoki K, Kim J, Guan KL. AMPK and mTOR in cellular energy homeostasis and drug targets. Annu Rev Pharmacol Toxicol 2012; 52: 381-400.

10. Laplante M, Sabatini DM. mTOR signaling in growth control and disease. Cell 2012; 149: 274-293.

11. Sarbassov DD, Ali SM, Sengupta $S$ et al. Prolonged rapamycin treatment inhibits mTORC2 assembly and Akt/PKB. Mol Cell 2006; 22: 159-168.

12. Lamming DW, Ye L, Katajisto $\mathrm{P}$ et al. Rapamycin-induced insulin resistance is mediated by mTORC2 loss and uncoupled from longevity. Science 2012; 335: 1638-1643.

13. Zinzalla V, Stracka D, Oppliger W, Hall MN. Activation of mTORC2 by association with the ribosome. Cell 2011; 144: 757768.

14. Han JM, Jeong SJ, Park MC et al. Leucyl-tRNA synthetase is an intracellular leucine sensor for the mTORC1-signaling pathway. Cell 2012; 149: 410-424.

15. Bonfils G, Jaquenoud M, Bontron S, Ostrowicz C, Ungermann C, De Virgilio C. Leucyl-tRNA synthetase controls TORC1 via the EGO complex. Mol Cell 2012; 46: 105-110.

16. Settembre C, Zoncu R, Medina DL et al. A lysosome-tonucleus signalling mechanism senses and regulates the lysosome via mTOR and TFEB. EMBO J 2012; 31: 1095-1108.

17. Bentzinger CF, Romanino K, Cloetta D et al. Skeletal musclespecific ablation of raptor, but not of rictor, causes metabolic changes and results in muscle dystrophy. Cell Metab 2008; 8: 411-424.

18. Um SH, Frigerio F, Watanabe M et al. Absence of S6K1 protects against age- and diet-induced obesity while enhancing insulin sensitivity. Nature 2004; 431: 200-205.

19. Khamzina L, Veilleux A, Bergeron S, Marette A. Increased activation of the mammalian target of rapamycin pathway in liver and skeletal muscle of obese rats: possible involvement in obesity-linked insulin resistance. Endocrinology 2005; 146: 14731481.

20. Drake JC, Alway SE, Hollander JM, Williamson DL. AICAR treatment for 14 days normalizes obesity-induced dysregulation of TORC1 signaling and translational capacity in fasted skeletal muscle. Am J Physiol Regul Integr Comp Physiol 2010; 299: R1546-R1554.

21. Ranieri SC, Fusco S, Panieri E et al. Mammalian life-span determinant p66shcA mediates obesity-induced insulin resistance. Proc Natl Acad Sci U S A 2010; 107: 13420-13425.

22. Sung MM, Koonen DP, Soltys CL, Jacobs RL, Febbraio M, Dyck JR. Increased CD36 expression in middle-aged mice contributes to obesity-related cardiac hypertrophy in the absence of cardiac dysfunction. J Mol Med 2011; 89: 459-469. 
23. Turdi S, Kandadi MR, Zhao J, Huff AF, Du M, Ren J. Deficiency in AMP-activated protein kinase exaggerates high fat diet-induced cardiac hypertrophy and contractile dysfunction. J Mol Cell Cardiol 2011; 50: 712-722.

24. Kim JE, Chen J. Regulation of peroxisome proliferatoractivated receptor-gamma activity by mammalian target of rapamycin and amino acids in adipogenesis. Diabetes 2004; 53: 2748-2756.

25. Zhang HH, Huang J, Duvel K et al. Insulin stimulates adipogenesis through the Akt-TSC2-mTORC1 pathway. Plos ONE 2009; 4: e6189.

26. Polak P, Cybulski N, Feige JN, Auwerx J, Ruegg MA, Hall $\mathrm{MN}$. Adipose-specific knockout of raptor results in lean mice with enhanced mitochondrial respiration. Cell Metab 2008; 8: 399 410.

27. Hagiwara A, Cornu M, Cybulski N et al. Hepatic mTORC2 activates glycolysis and lipogenesis through Akt, glucokinase, and SREBP1c. Cell Metab 2012; 15: 725-738.

28. Wang X, Hu Z, Hu J, Du J, Mitch WE. Insulin resistance accelerates muscle protein degradation: activation of the ubiquitinproteasome pathway by defects in muscle cell signaling. Endocrinology 2006; 147: 4160-4168.

29. Wannamethee SG, Shaper AG, Lennon L, Whincup PH. Decreased muscle mass and increased central adiposity are independently related to mortality in older men. Am J Clin Nutr 2007; 86: 1339-1346.

30. Williamson DL. Normalizing a hyperactive mTOR initiates muscle growth during obesity. Aging (Albany NY) 2011; 3: 8384.

31. Murakami M, Ichisaka T, Maeda M et al. mTOR is essential for growth and proliferation in early mouse embryos and embryonic stem cells. Mol Cell Biol 2004; 24: 6710-6718.

32. Pende M, Kozma SC, Jaquet M et al. Hypoinsulinaemia, glucose intolerance and diminished beta-cell size in S6K1-deficient mice. Nature 2000; 408: 994-997.

33. Briaud I, Dickson LM, Lingohr MK, McCuaig JF, Lawrence JC, Rhodes CJ. Insulin receptor substrate-2 proteasomal degradation mediated by a mammalian target of rapamycin (mTOR)induced negative feedback down-regulates protein kinase B-mediated signaling pathway in beta-cells. I Biol Chem 2005; 280: 2282-2293.

34. Harrison DE, Strong R, Sharp ZD et al. Rapamycin fed late in life extends lifespan in genetically heterogeneous mice. Nature 2009; 460: 392-395.

35. Miller RA, Harrison DE, Astle CM et al. Rapamycin, but not resveratrol or simvastatin, extends life span of genetically heterogeneous mice. J Gerontol A Biol Sci Med Sci 2011; 66: 191-201. 36. Majumder S, Caccamo A, Medina DX et al. Lifelong rapamycin administration ameliorates age-dependent cognitive deficits by reducing IL-1beta and enhancing NMDA signaling. Aging Cell 2012; 11: 326-335.

37. Holzenberger M, Dupont J, Ducos B et al. IGF-1 receptor regulates lifespan and resistance to oxidative stress in mice. Nature 2003; 421: 182-187.

38. Selman C, Lingard S, Choudhury AI et al. Evidence for lifespan extension and delayed age-related biomarkers in insulin receptor substrate 1 null mice. FASEB J 2008; 22: 807-818.

39. Fontana L, Partridge L, Longo VD. Extending healthy life span - from yeast to humans. Science 2010; 328: 321-326.

40. Colman RJ, Anderson RM, Johnson SC et al. Caloric restriction delays disease onset and mortality in rhesus monkeys. Science 2009; 325: 201-204.

41. Willcox BJ, Willcox DC, Todoriki $\mathrm{H}$ et al. Caloric restriction, the traditional Okinawan diet, and healthy aging: the diet of the world's longest-lived people and its potential impact on morbidity and life span. Ann N Y Acad Sci 2007; 1114: 434-455. 42. Fontana L, Meyer TE, Klein S, Holloszy JO. Long-term calorie restriction is highly effective in reducing the risk for atherosclerosis in humans. Proc Natl Acad Sci U S A 2004; 101: 66596663.

43. Meyer TE, Kovacs SJ, Ehsani AA, Klein S, Holloszy JO, Fontana L. Long-term caloric restriction ameliorates the decline in diastolic function in humans. I Am Coll Cardiol 2006; 47: 398402 .

44. Hammer S, Snel M, Lamb HJ et al. Prolonged caloric restriction in obese patients with type 2 diabetes mellitus decreases myocardial triglyceride content and improves myocardial function. J Am Coll Cardiol 2008; 52: 1006-1012.

45. Kapahi P, Chen D, Rogers AN et al. With TOR, less is more: a key role for the conserved nutrient-sensing TOR pathway in aging. Cell Metab 2010; 11: 453-465.

46. Zid BM, Rogers AN, Katewa SD et al. 4E-BP extends lifespan upon dietary restriction by enhancing mitochondrial activity in Drosophila. Cell 2009; 139: 149-160.

47. Cao K, Graziotto JJ, Blair CD et al. Rapamycin reverses cellular phenotypes and enhances mutant protein clearance in Hutchinson-Gilford progeria syndrome cells. Sci Transl Med 2011; 3: 89 ra58.

48. Castilho RM, Squarize CH, Chodosh LA, Williams BO, Gutkind JS. mTOR mediates Wnt-induced epidermal stem cell exhaustion and aging. Cell Stem Cell 2009; 5: 279-289.

49. Chen C, Liu Y, Liu Y, Zheng P. mTOR regulation and therapeutic rejuvenation of aging hematopoietic stem cells. Sci Signal 2009; 2: ra75.

50. Pulakat L, DeMarco VG, Ardhanari S et al. Adaptive mechanisms to compensate for overnutrition-induced cardiovascular abnormalities. Am J Physiol Regul Integr Comp Physiol 2011; 301: R885-R895.

51. Clavijo LC, Pinto TL, Kuchulakanti PK et al. Metabolic syndrome in patients with acute myocardial infarction is associated with increased infarct size and in-hospital complications. Cardiovasc Revasc Med 2006; 7: 7-11.

52. Mourmoura E, Leguen M, Dubouchaud $\mathrm{H}$ et al. Middle age aggravates myocardial ischemia through surprising upholding of complex II activity, oxidative stress, and reduced coronary perfusion. Age (Dordr) 2011; 33: 321-336.

53. Qin F, Siwik DA, Luptak I et al. The polyphenols resveratrol and S17834 prevent the structural and functional sequelae of diet-induced metabolic heart disease in mice. Circulation 2012; 125: 1757-1756.

54. Shih H, Lee B, Lee RJ, Boyle AJ. The aging heart and postinfarction left ventricular remodeling. J Am Coll Cardiol 2011; 57: 9-17.

55. North BJ, Sinclair DA. The intersection between aging and cardiovascular disease. Circ Res 2012; 110: 1097-1108.

56. Iozzo P. Myocardial, perivascular, and epicardial fat. Diabetes Care 2011; 34(Suppl. 2): S371-S379.

57. Olefsky JM, Glass CK. Macrophages, inflammation, and insulin resistance. Annu Rev Physiol 2010; 72: 219-246.

58. Wu D, Ren Z, Pae M et al. Aging up-regulates expression of inflammatory mediators in mouse adipose tissue. J Immunol 2007; 179: 4829-4839.

59. Starr ME, Evers BM, Saito H. Age-associated increase in cytokine production during systemic inflammation: adipose tissue as a major source of IL-6. J Gerontol A Biol Sci Med Sci 2009; 64: $723-730$.

60. Lakatta EG. Heart aging: a fly in the ointment? Circ Res 2001; 88: 984-986. 
61. Clifton PM, Keogh JB, Foster PR, Noakes M. Effect of weight loss on inflammatory and endothelial markers and FMD using two low-fat diets. Int J Obes (Lond) 2005; 29: 1445-1451.

62. Skurk T, Hauner H. Obesity and impaired fibrinolysis: role of adipose production of plasminogen activator inhibitor-1. Int $J$ Obes Relat Metab Disord 2004; 28: 1357-1364.

63. Samad F, Pandey M, Loskutoff DJ. Tissue factor gene expression in the adipose tissues of obese mice. Proc Natl Acad Sci U S A 1998; 95: 7591-7596.

64. Viswambharan H, Ming XF, Zhu S et al. Reconstituted highdensity lipoprotein inhibits thrombin-induced endothelial tissue factor expression through inhibition of RhoA and stimulation of phosphatidylinositol 3-kinase but not Akt/endothelial nitric oxide synthase. Circ Res 2004; 94: 918-925.

65. Franchini M, Targher G, Montagnana M, Lippi G. The metabolic syndrome and the risk of arterial and venous thrombosis. Thromb Res 2008; 122: 727-735.

66. Engbers MJ, van Hylckama Vlieg A, Rosendaal FR. Venous thrombosis in the elderly: incidence, risk factors and risk groups. J Thromb Haemost 2010; 8: 2105-2112.

67. Werner N, Kosiol S, Schiegl T et al. Circulating endothelial progenitor cells and cardiovascular outcomes. N Engl J Med 2005; 353: 999-1007.

68. Fadini GP, Miorin M, Facco M et al. Circulating endothelial progenitor cells are reduced in peripheral vascular complications of type 2 diabetes mellitus. J Am Coll Cardiol 2005; 45: 14491457.

69. Tousoulis D, Andreou I, Antoniades C, Tentolouris C, Stefanadis C. Role of inflammation and oxidative stress in endothelial progenitor cell function and mobilization: therapeutic implications for cardiovascular diseases. Atherosclerosis 2008; 201: 236-247. 70. Furukawa S, Fujita T, Shimabukuro M et al. Increased oxidative stress in obesity and its impact on metabolic syndrome. J Clin Invest 2004; 114: 1752-1761.

71. Kregel KC, Zhang HJ. An integrated view of oxidative stress in aging: basic mechanisms, functional effects, and pathological considerations. Am J Physiol Regul Integr Comp Physiol 2007; 292: R18-R36.

72. Yang Z, Ming XF. Recent advances in understanding endothelial dysfunction in atherosclerosis. Clin Med Res 2006; 4: 53-65. 73. Duplain H, Burcelin R, Sartori C et al. Insulin resistance, hyperlipidemia, and hypertension in mice lacking endothelial nitric oxide synthase. Circulation 2001; 104: 342-345.

74. Knowles JW, Reddick RL, Jennette JC, Shesely EG, Smithies $\mathrm{O}$, Maeda N. Enhanced atherosclerosis and kidney dysfunction in eNOS(-/-)Apoe(-/-) mice are ameliorated by enalapril treatment. J Clin Invest 2000; 105: 451-458.

75. Feng Q, Song W, Lu X et al. Development of heart failure and congenital septal defects in mice lacking endothelial nitric oxide synthase. Circulation 2002; 106: 873-879.

76. Desrois M, Clarke K, Lan C et al. Upregulation of eNOS and unchanged energy metabolism in increased susceptibility of the aging type 2 diabetic GK rat heart to ischemic injury. Am J Physiol Heart Circ Physiol 2010; 299: H1679-H1686.

77. Ming XF, Barandier C, Viswambharan $\mathrm{H}$ et al. Thrombin stimulates human endothelial arginase enzymatic activity via RhoA/ROCK pathway: implications for atherosclerotic endothelial dysfunction. Circulation 2004; 110: 3708-3714.

78. Rajapakse AG, Yepuri G, Carvas JM et al. Hyperactive S6K1 mediates oxidative stress and endothelial dysfunction in aging: inhibition by resveratrol. Plos ONE 2011; 6: e19237.

79. Elrod JW, Duranski MR, Langston W et al. eNOS gene therapy exacerbates hepatic ischemia-reperfusion injury in diabetes: a role for eNOS uncoupling. Circ Res 2006; 99: 78-85.
80. Takaya T, Hirata K, Yamashita $\mathrm{T}$ et al. A specific role for eNOS-derived reactive oxygen species in atherosclerosis progression. Arterioscler Thromb Vasc Biol 2007; 27: 1632-1637.

81. Forstermann U, Sessa WC. Nitric oxide synthases: regulation and function. Eur Heart J 2012; 33: 829-837.

82. Berkowitz DE, White R, Li D et al. Arginase reciprocally regulates nitric oxide synthase activity and contributes to endothelial dysfunction in aging blood vessels. Circulation 2003; 108: 2000-2006.

83. Antoniades C, Shirodaria C, Leeson P et al. Association of plasma asymmetrical dimethylarginine (ADMA) with elevated vascular superoxide production and endothelial nitric oxide synthase uncoupling: implications for endothelial function in human atherosclerosis. Eur Heart J 2009; 30: 1142-1150.

84. Chen CA, Wang TY, Varadharaj S et al. S-glutathionylation uncouples eNOS and regulates its cellular and vascular function. Nature 2010; 468: 1115-1118.

85. Zhang D, Contu R, Latronico MV et al. MTORC1 regulates cardiac function and myocyte survival through 4E-BP1 inhibition in mice. J Clin Invest 2010; 120: 2805-2816.

86. Shende P, Plaisance I, Morandi C et al. Cardiac raptor ablation impairs adaptive hypertrophy, alters metabolic gene expression, and causes heart failure in mice. Circulation 2011; 123: 1073-1082.

87. Soesanto W, Lin HY, Hu E et al. Mammalian target of rapamycin is a critical regulator of cardiac hypertrophy in spontaneously hypertensive rats. Hypertension 2009; 54: 13211327.

88. Zhang $\mathrm{P}, \mathrm{Hu} \mathrm{X}, \mathrm{Xu} \mathrm{X}$ et al. AMP activated protein kinasealpha2 deficiency exacerbates pressure-overload-induced left ventricular hypertrophy and dysfunction in mice. Hypertension 2008; 52: 918-924.

89. Muniyappa R, Montagnani M, Koh KK, Quon MJ. Cardiovascular actions of insulin. Endocr Rev 2007; 28: 463-491.

90. Kim JA, Jang HJ, Martinez-Lemus LA, Sowers JR. Activation of $\mathrm{mTOR} / \mathrm{p} 70 \mathrm{~S} 6$ kinase by ANG II inhibits insulin stimulated endothelial nitric oxide synthase and vasodilation. Am J Physiol Endocrinol Metab 2011; 302: E201-E208.

91. Sciarretta S, Zhai P, Shao D et al. Rheb is a critical regulator of autophagy during myocardial ischemia: pathophysiological implications in obesity and metabolic syndrome. Circulation 2012; 125: 1134-1146.

92. Di R, Wu X, Chang Z et al. S6K inhibition renders cardiac protection against myocardial infarction through PDK1 phosphorylation of Akt. Biochem J 2012; 441: 199-207.

93. Birse RT, Choi J, Reardon K et al. High-fat-diet-induced obesity and heart dysfunction are regulated by the TOR pathway in Drosophila. Cell Metab 2010; 12: 533-544.

94. Pearson KJ, Baur JA, Lewis KN et al. Resveratrol delays agerelated deterioration and mimics transcriptional aspects of dietary restriction without extending life span. Cell Metab 2008; 8: 157168.

95. Rajapakse AG, Ming XF, Carvas JM, Yang Z. The hexosamine biosynthesis inhibitor azaserine prevents endothelial inflammation and dysfunction under hyperglycemic condition through antioxidant effects. Am J Physiol Heart Circ Physiol 2009; 296: H815-H822.

96. Miyauchi H, Minamino T, Tateno K, Kunieda T, Toko H, Komuro I. Akt negatively regulates the in vitro lifespan of human endothelial cells via a p53/p21-dependent pathway. ЕMBO J 2004; 23: 212-220.

97. Camici GG, Steffel J, Amanovic I et al. Rapamycin promotes arterial thrombosis in vivo: implications for everolimus and zotarolimus eluting stents. Eur Heart J 2010; 31: 236-242. 
98. Ming XF, Rajapakse AG, Carvas JM, Ruffieux J, Yang Z. Opposing and uncoupling effects of mTOR and S6K1 in the regulation of endothelial tissue factor expression. FEBS Lett 2010, 584: 135-140.

99. Hatakeyama K, Asada Y, Marutsuka K, Sato Y, Kamikubo Y, Sumiyoshi A. Localization and activity of tissue factor in human aortic atherosclerotic lesions. Atherosclerosis 1997; 133: 213-219. 100. Tutar E, Ozcan M, Kilickap M et al. Elevated whole-blood tissue factor procoagulant activity as a marker of restenosis after percutaneous transluminal coronary angioplasty and stent implantation. Circulation 2003; 108: 1581-1584.
101. Luscher TF, Steffel J, Eberli FR et al. Drug-eluting stent and coronary thrombosis: biological mechanisms and clinical implications. Circulation 2007; 115: 1051-1058.

102. Inoue T, Croce K, Morooka T, Sakuma M, Node K, Simon DI. Vascular inflammation and repair: implications for reendothelialization, restenosis, and stent thrombosis. JACC Cardiovasc Interv 2011; 4: 1057-1066.

103. Ming XF, Rajapakse AG, Carvas JM, Ruffieux J, Yang Z. Inhibition of $56 \mathrm{~K} 1$ accounts partially for the anti-inflammatory effects of the arginase inhibitor L-norvaline. BMC Cardiovasc Disord 2009; 9: 12. 